\title{
CLASSIFYING CLOSED 2-ORBIFOLDS WITH EULER CHARACTERISTICS
}

\author{
WHITNEY DUVAL, JOHN SCHULTE, CHRISTOPHER SEATON \\ and BRADFORD TAYLOR \\ Department of Mathematics and Computer Science, Rhodes College, 2000 N. Parkway, \\ Memphis, TN 38112, USA \\ e-mails: \{whitney.duval, johnschulte1987,bradfordptaylor\}@gmail.com; seatonc@rhodes.edu
}

(Received 15 May 2009; revised 21 December 2009; accepted 25 June 2010)

\begin{abstract}
We determine the extent to which the collection of $\Gamma$-Euler-Satake characteristics classify closed 2-orbifolds. In particular, we show that the closed, connected, effective, orientable 2-orbifolds are classified by the $\Gamma$-Euler-Satake characteristics corresponding to free or free abelian $\Gamma$ and are not classified by those corresponding to any finite set of finitely generated discrete groups. These results demonstrate that the $\Gamma$-Euler-Satake characteristics corresponding to free abelian $\Gamma$ constitute new invariants of orbifolds. Similarly, we show that such a classification is neither possible for non-orientable 2-orbifolds nor for non-effective 2-orbifolds using any collection of groups $\Gamma$.
\end{abstract}

2010 Mathematics Subject Classification. Primary 57R20, 57S17; Secondary 22A22, 57P99.

1. Introduction. In a recent paper [9], Christopher Seaton and Carla Farsi introduced the $\Gamma$-sectors of an orbifold $Q$, a generalization of the inertia orbifold of $Q$ that is defined for any finitely generated discrete group $\Gamma$. In this context, the inertia orbifold (originally defined by Kawasaki in [10]; see also [1, 5]) corresponds to the case $\Gamma=\mathbb{Z}$; similarly, the $k$-multi-sectors of Chen and Ruan (see [1] or [5]) correspond to the case when $\Gamma=\mathbb{F}_{k}$ is the free group with $k$ generators.

The $\Gamma$-Euler-Satake characteristic of an orbifold $Q$, denoted $\chi_{\Gamma}^{E S}(Q)$, is defined to be the Euler-Satake characteristic of the $\Gamma$-sectors of $Q$. In [8], it is shown that several Euler characteristics that have been defined for orbifolds correspond to the $\Gamma$-EulerSatake characteristic for a specific choice of $\Gamma$. Hence, the $\Gamma$-sectors offer a framework in which to generalize the Euler characteristics of Bryan and Fulman (see [3]) and Tamanoi (see $[\mathbf{1 6}, \mathbf{1 7}])$ to closed orbifolds that are not necessarily global quotients. In particular, the stringy orbifold Euler characteristic defined for global quotients in [6] and for general orbifolds in [14] corresponds to the case $\Gamma=\mathbb{Z}^{2}$.

In this paper, we address the question of whether the $\Gamma$-EulerSatake characteristics classify closed, connected, two-dimensional orbifolds. The diffeomorphism-types of all closed 2-orbifolds are well known (see e.g. [18] or [2]). Here, however, we express this classification in a framework generalizing the familiar classification of closed 2-manifolds. An additional motivation of this investigation is to explore the extent to which the $\Gamma$-Euler-Satake characteristics constitute new invariants for orbifolds. Indeed, from their definition, the degree to which collections of the $\Gamma$ Euler-Satake characteristics depend on one another is unclear. We will see, however, 
that the characteristics corresponding to abelian $\Gamma$ are in some sense independent; the class of two-dimensional orientable orbifolds is sufficiently large to illustrate this fact. In this case, each $\Gamma$-Euler-Satake characteristic corresponds to the $\Gamma^{\prime}$-Euler-Satake characteristic for an abelain $\Gamma^{\prime}$. In the future, we will investigate classes of orbifolds that may indicate the differences between abelian and non-abelian $\Gamma$.

To simplify notation, for a closed orbifold $Q$, we define

$$
\chi_{(l)}^{E S}(Q)=\chi_{\mathbb{Z}^{l}}^{E S}(Q)
$$

Then for $l \geq 1$, these Euler characteristics correspond to the orbifold Euler characteristics defined for global quotients in [3] (note that our $\chi_{(l)}^{E S}(Q)$ corresponds to $\chi_{l+1}(M, G)$ in [3] when $Q$ is given by the action of a finite group $G$ on a manifold $M)$. It is observed in [8, Section 4.1] that $\chi_{(l)}^{E S}(Q)$ also corresponds to the EulerSatake characteristic of the $l$ th inertia orbifold of $Q$ and the Euler characteristic of the (underlying topological space of the) $l-1$ st inertia orbifold.

If $Q$ is an abelian orbifold (i.e. all isotropy groups of $Q$ are abelian), it is easy to see that

$$
\chi_{(l)}^{E S}(Q)=\chi_{\mathbb{F}_{l}}^{E S}(Q)
$$

where $\mathbb{F}_{l}$ denotes the free group with $l$ generators; in particular, this follows from Lemma 3.14 of this paper. Therefore, $\chi_{(l)}^{E S}(Q)$ is the Euler-Satake characteristic of the l-multi-sectors of $Q$ (see [1]).

Of primary interest will be the case of a closed, connected, effective, orientable 2-orbifold $Q$, for which the $\chi_{(l)}^{E S}(Q)$ will play a dominant role. Our first main result is a positive classification of these orbifolds using the $\chi_{(l)}^{E S}$.

THEOREM 1.1. Let $Q$ and $Q^{\prime}$ be closed, connected, effective, orientable 2-orbifolds such that $\chi_{(l)}^{E S}(Q)=\chi_{(l)}^{E S}\left(Q^{\prime}\right)$ for any infinite set of non-negative integers $l$. Then $Q$ and $Q^{\prime}$ are diffeomorphic.

It is well known (see e.g. [11]) that closed, connected, orientable, two-dimensional manifolds are completely characterized by their Euler characteristic. If $Q$ is a manifold, the $\Gamma$-Euler-Satake characteristic of $Q$ reduces to the usual Euler characteristic for any $\Gamma$. Hence, Theorem 1.1 constitutes a generalization of this result to orbifolds. However, this class of orbifolds is large enough to produce the following.

THEOREM 1.2. Let $N \geq 2$ be an integer and let $\mathfrak{G}$ be any finite collection of finitely generated discrete groups. Then there are distinct closed, connected, effective, orientable 2-orbifolds $Q_{1}, Q_{2}, \ldots, Q_{N}$ such that for each $\Gamma \in \mathfrak{G}$,

$$
\chi_{\Gamma}^{E S}\left(Q_{1}\right)=\chi_{\Gamma}^{E S}\left(Q_{2}\right)=\cdots=\chi_{\Gamma}^{E S}\left(Q_{N}\right) .
$$

It follows that the classification of Theorem 1.1 cannot be improved upon using the $\Gamma$-Euler-Satake characteristics. Note that Theorem 3.13 is a slightly more general version of Theorem 1.2, though clumsier to state.

The outline of this work is as follows. In Section 2, we recall the necessary definitions and summarize the pertinent preliminary material. We study effective, orientable 2-orbifolds in Section 3 and prove Theorems 1.1 and 1.2. In Section 4, we demonstrate through examples that the hypotheses of Theorem 1.1 cannot be relaxed. 
2. Background and definitions. In this section, we briefly introduce the required definitions and fix notation. For a more thorough background on orbifolds, the reader is referred to [1] or [4]; see also $[\mathbf{2}, \mathbf{1 2}]$ or $[\mathbf{1 8}]$, and note that the orbifolds in these latter references correspond to effective orbifolds. We will have the occasion to consider non-effective orbifolds only in Example 4.1 and only in the form of a global quotient.

An orbifold $Q$ is most succinctly defined to be a Morita equivalence class of orbifold groupoids, i.e. proper étale Lie groupoids. Such a groupoid $\mathcal{G}$ is called a presentation of the orbifold $Q$, and two orbifold groupoids $\mathcal{G}$ and $\mathcal{G}^{\prime}$ present the same orbifold if and only if they are Morita equivalent. In this case, their orbit spaces $|\mathcal{G}|$ and $\left|\mathcal{G}^{\prime}\right|$ are naturally homeomorphic, and we say that they are diffeomorphic as orbifolds.

Fix a proper étale Lie groupoid $\mathcal{G}$ with space of objects $G_{0}$ and space of arrows $G_{1}$. For each $x \in G_{0}$, there is a neighbourhood $V_{x} \subseteq G_{0}$ of $x$ diffeomorphic to $\mathbb{R}^{n}$ such that if $G_{x}$ denotes the isotropy group of $x$, then there is a $G_{x}$-action on $V_{x}$, and the restriction $\left.\mathcal{G}\right|_{V_{x}}$ is isomorphic as a Lie groupoid to the translation groupoid $G_{x} \ltimes V_{x}$. We let $\pi_{x}: V_{x} \rightarrow|\mathcal{G}|$ denote the quotient map into the orbit space of $\mathcal{G}$. In this way, the definition of an orbifold in terms of orbifold charts is recovered, as $\left\{V_{x}, G_{x}, \pi_{x}\right\}$ gives an orbifold chart for $Q$ near the point representing the orbit of $x$. Note that we can always take $x$ to correspond to the origin in $\mathbb{R}^{n}$ and $G_{x}$ to act linearly; we then refer to $\left\{V_{x}, G_{x}, \pi_{x}\right\}$ as a linear chart. If $y$ is another point in $G_{0}$ in the orbit of $x$, then $G_{y}$ and $G_{x}$ are isomorphic. Hence, if $p \in|\mathcal{G}|$ denotes the orbit of $x$, then we can define $G_{p}$ to be (the isomorphism class of) $G_{x}$. The point $p \in|\mathcal{G}|$ is a non-singular point if $G_{p}$ is trivial and a singular point otherwise.

We say that the orbifold $Q$ is effective if $\mathcal{G}$ is an effective groupoid, or equivalently if the local $G_{x}$-actions on the $V_{x}$ are effective. The orbifold $Q$ is closed if it does not have boundary as an orbifold, i.e. $G_{0}$ is a manifold without boundary, and the orbit space $|\mathcal{G}|$ is a compact topological space. By connected, we mean that $|\mathcal{G}|$ is a connected topological space. The orbifold is oriented if $G_{0}$ is equipped with a $G_{1}$ invariant orientation; if $\mathcal{G}$ admits an orientation, we say that $Q$ is orientable. Note that each of these qualities is preserved under Morita equivalence so that they describe the orbifold $Q$ as well as the presentation $\mathcal{G}$.

If $Q$ is a closed, connected, effective, two-dimensional orbifold and $x \in G_{0}$, then $G_{x}$ is a finite subgroup of $O(2)$ (with respect to any inner product on $V_{x}$ ). It follows that $G_{x}$ is either a cyclic group acting as rotations, a group isomorphic to $\mathbb{Z} / 2 \mathbb{Z}$ acting as reflection through a line, or a group isomorphic to a dihedral group whose action is generated by reflections through two lines (see [18]). The singular points associated to these actions are referred to as cone points (or elliptic points), reflector lines and corner reflectors, respectively. Only the first of these three preserves an orientation of $\mathbb{R}^{2}$; hence, if we assume further that $Q$ is orientable, then the singular points are isolated cone points with cyclic isotropy. By the order of the cone point, we will mean the order of the isotropy group. It follows that the underlying space is homeomorphic to a closed, connected orientable surface, and the set of singular points corresponds to a finite collection $\left\{p_{1}, p_{2}, \ldots, p_{k}\right\}$ of cone points.

A closed, connected, effective, orientable, two-dimensional orbifold, then, is determined by the genus $g$ of the underlying space, a non-negative integer $k$ indicating the number of cone points and an integer $m_{i} \geq 2$ for $i=1,2, \ldots, k$ with $m_{1} \leq m_{2} \leq \cdots \leq m_{k}$, indicating the order of each cone point. We will use the notation $\Sigma_{g}\left(m_{1}, \ldots, m_{k}\right)$ to denote this orbifold. Note that we will often refer to the genus of the underlying space of $Q$ simply as the genus of $Q$. 
Let $Q$ be an orbifold. For each finitely generated discrete group $\Gamma$, we associate to $Q$ an orbifold $\tilde{Q}_{\Gamma}$ called the $\Gamma$-sectors of $Q$. We recall this construction briefly (see [9] for more details). Let $\mathcal{S}_{\mathcal{G}}^{\Gamma}$ denote the space of groupoid homomorphisms from $\Gamma$ into $\mathcal{G}$ or equivalently group homomorphisms from $\Gamma$ into an isotropy group $G_{x}$ of $\mathcal{G}$. Then $\mathcal{S}_{\mathcal{G}}^{\Gamma}$ has the structure of a smooth manifold, possibly with connected components of different dimensions. There is a natural $\mathcal{G}$-action on $\mathcal{S}_{\mathcal{G}}^{\Gamma}$ by pointwise conjugation, and the groupoid $\mathcal{G}^{\Gamma}=\mathcal{G} \ltimes \mathcal{S}_{\mathcal{G}}^{\Gamma}$ is a presentation of the orbifold of $\Gamma$-sectors $\tilde{Q}_{\Gamma}$. If $\left\{V_{x}, G_{x}, \pi_{x}\right\}$ is a linear chart for $Q$ and $\phi_{x}: \Gamma \rightarrow G_{x}$ is a homomorphism, then $\left\{V_{x}^{\left\langle\phi_{x}\right\rangle}, C_{G_{x}}\left(\phi_{x}\right), \pi_{x}^{\phi_{x}}\right\}$ is a linear chart for $\tilde{Q}_{\Gamma}$ near $\phi_{x}$, where $V_{x}^{\left\langle\phi_{x}\right\rangle}$ denotes the subspace of $V_{x}$ fixed by the image of $\phi_{x}, C_{G_{x}}\left(\phi_{x}\right)$ is the centralizer of the image of $\phi_{x}$ in $G_{x}$, and $\pi_{x}^{\phi_{x}}$ denotes the quotient map of the $C_{G_{x}}\left(\phi_{x}\right)$-action. The connected component $\tilde{Q}_{(1)}$ of $\tilde{Q}_{\Gamma}$ corresponding to the identity homomorphism (into any isotropy group) is diffeomorphic to $Q$. We denote the connected component of a homomorphism $\phi_{x}: \Gamma \rightarrow G_{x}$ by $\tilde{Q}_{(\phi)}$. Note that the $\mathbb{Z}$-sectors correspond to the inertia orbifold, and the $\mathbb{F}_{l}$-sectors correspond to the l-multi-sectors (see [7]; see also [1] or [5] for the definitions).

In the case that $Q$ is presented by $M \rtimes G$ where $G$ is a finite group acting on the smooth manifold $M$, then our description of the $\Gamma$-sectors corresponds to that of Tamanoi in $[\mathbf{1 6}, \mathbf{1 7}]$, where

$$
\tilde{Q}_{\Gamma}=\coprod_{(\phi) \in \operatorname{HOM}(\Gamma, G) / G} M^{\langle\phi\rangle} \rtimes C_{G}(\phi) .
$$

Here, the union is over conjugacy classes $(\phi)$ of homomorphisms $\phi \in \operatorname{HOM}(\Gamma, G)$. In this case, we use $(M ; G)_{(\phi)}$ to denote $M^{\langle\phi\rangle} \rtimes C_{G}(\phi)$. Note that this description coincides with ours more generally for $G$ a Lie group with certain restrictions on the action (see [7, Section 3]).

The Euler-Satake characteristic was first defined in [15], then called the Euler characteristic as a $V$-manifold. Satake's definition generalizes directly to the noneffective case. Given a simplicial decomposition $\mathcal{T}$ of the underlying space of $Q$ such that the order of the isotropy group $G_{\sigma}$ on the interior of each simplex $\sigma \in \mathcal{T}$ is constant (which always exists; see [13] or [8]), we define

$$
\chi_{E S}(Q)=\sum_{\sigma \in \mathcal{T}} \frac{(-1)^{\operatorname{dim} \sigma}}{\left|G_{\sigma}\right|} .
$$

The Euler-Satake characteristic clearly reduces to the usual Euler characteristic in the case that each isotropy group of $Q$ is trivial, i.e. in the case of a manifold.

Given a finitely generated discrete group, we define the $\Gamma$-Euler-Satake characteristic of $Q$ to be the Euler-Satake characteristic of the $\Gamma$-sectors of $Q$; i.e.

$$
\chi_{\Gamma}^{E S}(Q)=\chi_{E S}\left(\tilde{Q}_{\Gamma}\right)
$$

The Euler-Satake characteristic of a disconnected orbifold is of course equal to the sum of the Euler-Satake characteristics of the connected components. See [8] for properties of the Euler-Satake characteristic and $\Gamma$-Euler-Satake characteristics.

3. The $\Gamma$-Euler-Satake characteristics of effective, orientable 2 -orbifolds. In this section, we restrict our attention to closed, connected, effective, orientable 2-orbifolds. 
In Section 3.1, we determine a formula for the $l$ th Euler-Satake characteristics in this case and use this formula to prove Theorem 1.1. In Section 3.2, we construct for each finite collection of non-negative integers $l$ an arbitrarily large (finite) collection of orbifolds such that the $l$ th Euler-Satake characteristics coincide. In Section 3.3, we generalize to arbitrary $\Gamma$, proving Theorem 1.2.

3.1. The classification for free Abelian $\Gamma$. Let $Q$ be a closed, connected, effective, orientable 2-orbifold. As mentioned in Section 2, $Q$ is of the form $\Sigma_{g}\left(m_{1}, \ldots, m_{k}\right)$ for some non-negative integers $g$ and $k$ and integers $2 \leq m_{1} \leq m_{2} \leq \cdots \leq m_{k}$. Let $\mathcal{G}$ be an orbifold groupoid presenting $Q$. We begin by describing $\tilde{Q}_{\Gamma}$ in this case.

Given a finitely generated discrete group $\Gamma$, a homomorphism $\phi_{x}: \Gamma \rightarrow \mathcal{G}$ corresponds to a choice of a point $x$ in an orbifold chart $\left\{V_{x}, G_{x}, \pi_{x}\right\}$ for $Q$ and a homomorphism $\Gamma \rightarrow G_{x}$, which we also denote $\phi_{x}$. If $\phi_{x}$ is trivial so that its image is the trivial group, then it is on the same connected component as all such homomorphisms, and $\tilde{Q}_{(\phi)}$ is diffeomorphic to $Q$. Otherwise, $\pi_{x}(x)=p_{i}$ is one of the singular points of $Q$, and $\phi_{x}$ corresponds to a non-trivial homomorphisms into $\mathbb{Z} / m_{i} \mathbb{Z}$ acting on $V_{x}=\mathbb{R}^{2}$ by rotations. It follows that the ( $\operatorname{Im} \phi_{x}$ )-fixed-point subset of $\mathbb{R}^{2}$ consists of a single point $x$, and $\phi_{x}$ is the only point in the connected component $\tilde{Q}_{(\phi)}$ of $\tilde{Q}_{\Gamma}$. A chart for $\tilde{Q}_{(\phi)}$ is of the form $\left\{V_{x}^{\left\langle\phi_{x}\right\rangle}, C_{G}\left(\phi_{x}\right), \pi_{x}^{\phi_{x}}\right\}=\left\{\{x\}, \mathbb{Z} / m_{i} \mathbb{Z}, \pi_{x}^{\phi_{x}}\right\}$, so that $\tilde{Q}_{(\phi)}$ is a point equipped with the trivial action of $\mathbb{Z} / m_{i} \mathbb{Z}$. As the local groups of $Q$ are abelian, and as the singular points of $Q$ are isolated, the $\mathcal{G}$-orbits of non-trivial homomorphisms $\phi_{x}$ are trivial. Hence, for each cone point $p_{i}$ with isotropy group $\mathbb{Z} / m_{i} \mathbb{Z}$, there are exactly $\left|\operatorname{HOM}\left(\Gamma, \mathbb{Z} / m_{i} \mathbb{Z}\right)\right|-1$ connected components corresponding to $p_{i}$ with trivial $\mathbb{Z} / m_{i} \mathbb{Z}$-action.

We use these observations to derive the following, which gives a formula for the $l$ th Euler-Satake characteristic of a closed, connected, effective, orientable 2-orbifold.

Proposition 3.1. Let $Q=\Sigma_{g}\left(m_{1}, \ldots, m_{k}\right)$ be a closed, connected, effective, orientable 2-orbifold with notation as above. Then for each integer $l \geq 0$,

$$
\chi_{(l)}^{E S}(Q)=2-2 g-k+\sum_{i=1}^{k} m_{i}^{l-1} .
$$

Proof. Let $\mathcal{T}$ be a simplicial decomposition of $Q$ subordinate to the singular strata (see [13] or [7]); in this context, this means simply that each singular point $p_{i}$ corresponds to a vertex of $\mathcal{T}$. Then

$$
\begin{aligned}
\sum_{\sigma \in \mathcal{T}}(-1)^{\operatorname{dim} \sigma} & =\chi_{t o p}(Q) \\
& =2-2 g,
\end{aligned}
$$

where $\chi_{t o p}(Q)$ denotes the usual Euler characteristic of the underlying space of $Q$. It follows that

$$
\begin{aligned}
\chi_{(0)}^{E S}(Q) & =\chi_{E S}(Q) \\
& =\sum_{\sigma \in \mathcal{T}}(-1)^{\operatorname{dim} \sigma}-k+\sum_{i=1}^{k} \frac{1}{m_{i}} \\
& =2-2 g-k+\sum_{i=1}^{k} \frac{1}{m_{i}} .
\end{aligned}
$$


Now, let $l \geq 0$ be an integer. Each cone point $p_{i}$ of order $m_{i}$ corresponds to $\left|\operatorname{HOM}\left(\mathbb{Z}^{l}, \mathbb{Z} / m_{i} \mathbb{Z}\right)\right|-1=m_{i}^{l}-1$ identical $\mathbb{Z}^{l}$-sectors, each given by a single point equipped with the trivial action of $\mathbb{Z} / m_{i} \mathbb{Z}$. It follows that the Euler-Satake characteristic of the corresponding $\Gamma$-sector is $\frac{1}{m_{i}}$, and hence

$$
\begin{aligned}
\chi_{(l)}^{E S}(Q) & =2-2 g-k+\sum_{i=1}^{k} \frac{1}{m_{i}}+\sum_{i=1}^{k}\left(m_{i}^{l}-1\right) \frac{1}{m_{i}} \\
& =2-2 g-k+\sum_{i=1}^{k} m_{i}^{l-1}
\end{aligned}
$$

completing the proof.

Note that as $\chi_{(0)}^{E S}(Q)=\chi_{E S}(Q)$, the case $l=0$ of equation (2) coincides with [18, Equation 13.3.4] for orientable orbifolds (which do not have corner reflectors).

It is easy to see that distinct 2-orbifolds may have the same Euler-Satake characteristic even when they have homeomorphic underlying spaces, as illustrated with the following.

EXAMPLE 3.2. Let $g \geq 0$ be an integer and $Q$ the orbifold with underlying space $\Sigma_{g}$ and nine cone points, each of order 3 . Let $Q^{\prime}$ be the orbifold with underlying space $\Sigma_{g}$ and eight cone points, each of order 4 . Then

$$
\begin{aligned}
\chi_{E S}(Q) & =-4-2 g \\
& =\chi_{E S}\left(Q^{\prime}\right) .
\end{aligned}
$$

However, there can be only finitely many orbifolds with the same Euler-Satake characteristic.

LEMMA 3.3. Let $Q$ be a closed, connected, effective, orientable 2-orbifold of genus $g$. Then there are only finitely many closed, connected, effective, orientable 2-orbifolds with the same Euler-Satake characteristic.

Proof. We let $Q=\Sigma_{g}\left(m_{1}, \ldots, m_{k}\right)$ as above and $m=m_{k}=\max _{i=1, \ldots, k} m_{i}$. Then

$$
\begin{aligned}
2-2 g-k+\frac{k}{m} & =\frac{(2-2 g-k) m+k}{m} \\
& \leq \chi_{E S}(Q) .
\end{aligned}
$$

Let $Q^{\prime}=\Sigma_{g^{\prime}}\left(m_{1}^{\prime}, \ldots, m_{k^{\prime}}^{\prime}\right)$ be another orbifold such that $\chi_{E S}(Q)=\chi_{E S}\left(Q^{\prime}\right)$. Then as each $m_{i}^{\prime} \geq 2$,

$$
\begin{aligned}
\frac{(2-2 g-k) m+k}{m} & \leq \chi_{E S}\left(Q^{\prime}\right) \\
& =2-2 g^{\prime}-k^{\prime}+\sum_{i=1}^{k^{\prime}} \frac{1}{m_{i}^{\prime}} \\
& \leq 2-2 g^{\prime}-\frac{k^{\prime}}{2} \\
& \leq 2-2 g^{\prime} .
\end{aligned}
$$


It follows that

$$
g^{\prime} \leq g+\frac{k(m-1)}{2 m},
$$

implying that there are only a finite number of possible values of $g^{\prime} \geq 0$. Using the estimate $[(2-2 g-k) m+k] / m \leq 2-2 g^{\prime}-k^{\prime} / 2$ from equation (3), it follows that

$$
k^{\prime} \leq 4 g-4 g^{\prime}+\frac{2 k(m-1)}{m},
$$

implying that for each possible value of $g^{\prime}$, there is a finite number of possible values of $k^{\prime} \geq 0$.

To complete the proof, we fix values of $g^{\prime}$ and $k^{\prime}$ and show that there are a finite number of possibilities for the orders $m_{1}^{\prime}, \ldots, m_{k^{\prime}}^{\prime}$. Note that if $k^{\prime}=0$, there is nothing to prove, so assume $k^{\prime}>0$. Without loss of generality, we assume as above that $m_{1}^{\prime} \leq m_{2}^{\prime} \leq \cdots \leq m_{k^{\prime}}^{\prime}$. As $\chi_{E S}(Q)=\chi_{E S}\left(Q^{\prime}\right)$, we have

$$
2\left(g^{\prime}-g\right)+k^{\prime}-k+\sum_{i=1}^{k} \frac{1}{m_{i}}=\sum_{i=1}^{k^{\prime}} \frac{1}{m_{i}^{\prime}} .
$$

Let $C_{1}=2\left(g^{\prime}-g\right)+k^{\prime}-k+\sum_{i=1}^{k} \frac{1}{m_{i}}$ denote the left side of this equation. If $C_{1} \leq 0$, then as $k^{\prime}>0$, there are no solutions for the $m_{i}^{\prime}$, so assume that $C_{1}>0$. Then as

$$
C_{1}=\sum_{i=1}^{k^{\prime}} \frac{1}{m_{i}^{\prime}} \leq \frac{k^{\prime}}{m_{1}^{\prime}}
$$

we have that

$$
m_{1}^{\prime} \leq \frac{k^{\prime}}{C_{1}},
$$

and hence that there is a finite number of possibilities for $m_{1}^{\prime}$. Fixing one such $m_{1}^{\prime}$ and returning to equation (4), we let $C_{2}=2\left(g^{\prime}-g\right)+k^{\prime}-k-\frac{1}{m_{1}^{\prime}}+\sum_{i=1}^{k} \frac{1}{m_{i}}$ and then

$$
C_{2}=\sum_{i=2}^{k^{\prime}} \frac{1}{m_{i}^{\prime}} \leq \frac{k^{\prime}-1}{m_{2}^{\prime}} .
$$

Hence either $C_{2} \leq 0$, yielding no solution for $m_{2}^{\prime}, \ldots, m_{k^{\prime}}^{\prime}$, or

$$
m_{2}^{\prime} \leq \frac{k^{\prime}-1}{C_{2}} .
$$

It follows that there is a finite number of possibilities for $m_{2}^{\prime}$.

Continuing in the same way, we see that given values of $m_{1}^{\prime}, \ldots, m_{i}^{\prime}$ for some $i<k^{\prime}$, there is a finite number of possibilities for $m_{i+1}^{\prime}$, completing the proof. instance,

This is no longer the case for the higher Euler-Satake characteristics $\chi_{(l)}^{E S}$. For

$$
\chi_{(1)}^{E S}(Q)=2-2 g
$$


coincides with the usual Euler characteristic of the underlying space (note that this is the case in arbitrary dimension; see [8]). It follows that this characteristic coincides for any orbifolds with the same underlying space. For $l>1$, infinite families of orbifolds whose $l$ th Euler-Satake characteristics coincide can be constructed.

EXAMPLE 3.4. Fix integers $j, l \geq 2$ with $j$ odd. For each odd integer $k \geq 1$, the orbifold $Q_{k}$ of genus $g_{k}=\frac{1}{2} k\left(j^{l-1}-1\right)$ with $k$ cone points, each of order $j$, satisfies

$$
\begin{aligned}
\chi_{(l)}^{E S}\left(Q_{k}\right) & =2-2 g_{k}-k+j^{l-1} k \\
& =2 .
\end{aligned}
$$

It is clear, then, that none of the $l$ th Euler-Satake characteristics classify this class of 2-orbifolds. However, as stated in Theorem 1.1, any infinite collection of the $l$ th Euler-Satake characteristics is sufficient for classifying this class of orbifolds. We have the following technical result before proceeding to the proof of Theorem 1.1.

LeMma 3.5. Let $\mathfrak{L}$ be a set of non-negative integers. Suppose $Q$ and $Q^{\prime}$ are closed, connected, effective, orientable 2-orbifolds such that

$$
\chi_{(l)}^{E S}(Q)=\chi_{(l)}^{E S}\left(Q^{\prime}\right)
$$

for $l \in \mathfrak{L}$. Suppose $Q$ and $Q^{\prime}$ both have at least one cone point of order $m$. If $\mathcal{Q}$ is the orbifold formed by removing a cone point of order $m$ from $Q$ and $\mathcal{Q}^{\prime}$ the orbifold formed by removing a cone point of order $m$ from $Q^{\prime}$, then

$$
\chi_{(l)}^{E S}(\mathcal{Q})=\chi_{(l)}^{E S}\left(\mathcal{Q}^{\prime}\right)
$$

for $l \in \mathfrak{L}$.

Proof. We simply note that

$$
\begin{aligned}
\chi_{(l)}^{E S}(\mathcal{Q}) & =\chi_{(l)}^{E S}(Q)+1-m^{l-1} \\
& =\chi_{(l)}^{E S}\left(Q^{\prime}\right)+1-m^{l-1} \\
& =\chi_{(l)}^{E S}\left(\mathcal{Q}^{\prime}\right)
\end{aligned}
$$

for each $l \in \mathfrak{L}$.

Proof of Theorem 1.1. Assume $Q$ and $Q^{\prime}$ are distinct, connected, effective, orientable 2-orbifolds such that $\chi_{(l)}^{E S}(Q)=\chi_{(l)}^{E S}\left(Q^{\prime}\right)$ for every $l$ in some infinite set $\mathfrak{L}$ of non-negative integers. Let $Q=\Sigma_{g}\left(m_{1}, \ldots, m_{k}\right)$ and $Q^{\prime}=\Sigma_{g^{\prime}}\left(m_{1}^{\prime}, \ldots, m_{k \prime}^{\prime}\right)$ as above. If $k=0$ or $k^{\prime}=0$, then the result is trivial, so assume not. By Lemma 3.5, we can assume without loss of generality that $m_{k}>m_{k^{\prime}}^{\prime}$.

For each $l \in \mathfrak{L}$, we have by Proposition 3.1 that

$$
2-2 g-k+\left(\sum_{i=1}^{k} m_{i}^{l-1}\right)=2-2 g^{\prime}-k^{\prime}+\left(\sum_{i=1}^{k^{\prime}}\left(m_{i}^{\prime}\right)^{l-1}\right)
$$


Noting that the right-hand side is zero for at most one value of $l$, it follows that for $l \in \mathfrak{L}$ sufficiently large,

$$
\frac{2-2 g-k+\left(\sum_{i=1}^{k} m_{i}^{l-1}\right)}{2-2 g^{\prime}-k^{\prime}+\left(\sum_{i=1}^{k^{\prime}}\left(m_{i}^{\prime}\right)^{l-1}\right)}=1 .
$$

Based on the order relationships between the $m_{i}$ and $m_{i}^{\prime}$, we have that

$$
\begin{aligned}
\frac{2-2 g-k+\left(\sum_{i=1}^{k} m_{i}^{l-1}\right)}{2-2 g^{\prime}-k^{\prime}+\left(\sum_{i=1}^{k^{\prime}}\left(m_{i}^{\prime}\right)^{l-1}\right)} & \geq \frac{\left(\sum_{i=1}^{k} m_{i}^{l-1}\right)-k-2 g}{2+\sum_{i=1}^{k^{\prime}}\left(m_{i}^{\prime}\right)^{l-1}} \\
& \geq \frac{m_{k}^{l-1}-k-2 g}{\left(m_{k^{\prime}}^{\prime}\right)^{l-1}+k^{\prime}\left(m_{k^{\prime}}^{\prime}\right)^{l-1}} \\
& =\frac{m_{k}^{l-1}}{\left(k^{\prime}+1\right)\left(m_{k^{\prime}}^{\prime}\right)^{l-1}-\frac{k+2 g}{\left(k^{\prime}+1\right)\left(m_{k^{\prime}}^{\prime}\right)^{l-1}}} \\
& =\frac{1}{k^{\prime}+1}\left(\frac{m_{k}}{m_{k^{\prime}}^{\prime}}\right)^{l-1}-\frac{k+2 g}{\left(k^{\prime}+1\right)\left(m_{k^{\prime}}^{\prime}\right)^{l-1}} .
\end{aligned}
$$

However, as $m_{k}>m_{k^{\prime}}^{\prime}$, it follows that

$$
\lim _{l \rightarrow \infty} \frac{1}{k^{\prime}+1}\left(\frac{m_{k}}{m_{k^{\prime}}^{\prime}}\right)^{l-1}-\frac{k+2 g}{\left(k^{\prime}+1\right)\left(m_{k^{\prime}}^{\prime}\right)^{l-1}}=\infty,
$$

a contradiction. It follows that $Q=Q^{\prime}$.

3.2. Negative classification results for $\Gamma$ free abelian. In this section, we demonstrate that Theorem 1.1 cannot be improved upon in the case of closed, connected, effective, orientable 2-orbifolds. For any finite collection of the $l$ th EulerSatake characteristics, we construct an arbitrarily large (finite) collection of orbifolds whose $l$ th Euler-Satake characteristics coincide. Specifically, the goal of this section is to prove the following, which will be used to prove Theorem 1.2 and Theorem 3.13. In particular, the perhaps mysterious conditions of the orders of the cone points imposed throughout this section will allow us to extend to the $\Gamma$-Euler-Satake characteristics for arbitrary $\Gamma$ in Section 3.3.

Proposition 3.6. Let $L \geq 0$ and $N \geq 1$ be integers. Then there are $N$ distinct closed, connected, effective, orientable 2-orbifolds $Q_{1}, Q_{2}, Q_{3}, \ldots, Q_{N}$ such that for each $l=$ $0,1, \ldots, L$,

$$
\chi_{(l)}^{E S}\left(Q_{1}\right)=\chi_{(l)}^{E S}\left(Q_{2}\right)=\cdots=\chi_{(l)}^{E S}\left(Q_{N}\right)
$$


The common genus of these orbifolds can be taken to be any non-negative integer $g$. Moreover, if $R$ is any collection of $2^{L-2}$ integers $\geq 2$, then the orders of the cone points of the $Q_{j}$ can be taken to be elements of the set $\left\{2 q+1,2 q^{2}+q, q+2,2 q+q^{2}: q \in R\right\}$.

First, we establish a number of results and constructions that will simplify the arguments and notation in this section.

Definition 3.7. Let $Q=\Sigma_{g}\left(m_{1}, \ldots, m_{k}\right)$ and $Q^{\prime}=\Sigma_{g}\left(m_{1}^{\prime}, \ldots, m_{k^{\prime}}^{\prime}\right)$ be two orbifolds with the same genus. For any integer $s \geq 1$, we let

$$
s \diamond Q=\Sigma_{g}\left(s m_{1}, \ldots, s m_{k}\right)
$$

denote the orbifold with the same genus and number of cone points as $Q$ such that the order of each cone point is multiplied by $s$. For any integer $t \geq 1$, we let

$$
t \star Q=\Sigma_{g}(\overbrace{m_{1}, \ldots, m_{1}}^{t}, \overbrace{m_{2}, \ldots, m_{2}}^{t}, \ldots, \overbrace{m_{k}, \ldots, m_{k}}^{t})
$$

denote the orbifold with the same genus as $Q$ and each cone point of $Q$ occurring $t$ times. We let

$$
Q \circledast Q^{\prime}=\Sigma_{g}\left(m_{1}, \ldots, m_{k}, m_{1}^{\prime}, \ldots, m_{k^{\prime}}^{\prime}\right)
$$

denote the orbifold with the same genus as $Q$ and $Q^{\prime}$ and the combined $k+k^{\prime}$ cone points of both $Q$ and $Q^{\prime}$.

Note that $\circledast$ is clearly commutative and associative, and

$$
t \star Q=\overbrace{Q \circledast \cdots \circledast Q}^{t} .
$$

Moreover, $1 \star Q=1 \diamond Q=Q$. In the case that the genus of $Q$ and $Q^{\prime}$ is zero, $Q \circledast Q^{\prime}$ corresponds to the connected sum (defined in the same way as manifolds with the additional assumption that the disks removed contain no singular points) so that $t \star Q$ corresponds to the $t$-fold connected sum of $Q$ with itself.

Lemma 3.8. Let $L, s$ and $t$ be non-negative integers. Suppose $Q$ and $Q^{\prime}$ are closed, connected, effective, orientable 2-orbifolds with the same number of cone points such that

$$
\chi_{(l)}^{E S}(Q)=\chi_{(l)}^{E S}\left(Q^{\prime}\right)
$$

for $l \leq L$. Then if $Q$ and $Q^{\prime}$ have the same genus,

$$
\chi_{(l)}^{E S}(t \star Q)=\chi_{(l)}^{E S}\left(t \star Q^{\prime}\right)
$$

and

$$
\chi_{(l)}^{E S}(s \diamond Q)=\chi_{(l)}^{E S}\left(s \diamond Q^{\prime}\right)
$$

for each $l \leq L$.

Proof. Assume $Q=\Sigma_{g}\left(m_{1}, \ldots, m_{k}\right)$ and $Q^{\prime}=\Sigma_{g}\left(m_{1}^{\prime}, \ldots, m_{k}^{\prime}\right)$. The result then follows from direct computations and application of Proposition 3.1. 
Lemma 3.9. Let L be a non-negative integer, and let $Q_{1}, Q_{1}^{\prime}, \ldots, Q_{N}, Q_{N}^{\prime}$ be closed, connected, effective 2-orbifolds. Assume that for each $j=1, \ldots, N, Q_{j}$ and $Q_{j}^{\prime}$ have the same genus and number of cone points, and

$$
\chi_{(l)}^{E S}\left(Q_{j}\right)=\chi_{(l)}^{E S}\left(Q_{j}^{\prime}\right)
$$

for each $l \leq L$. Then there are closed, connected, effective, orientable 2-orbifolds $\mathcal{Q}_{1}, \mathcal{Q}_{1}^{\prime}, \ldots, \mathcal{Q}_{N}, \mathcal{Q}_{N}^{\prime}$ all with the same number of cone points such that for each $j=1, \ldots N$,

$$
\mathcal{Q}_{j}=t_{j} \star Q_{j}
$$

and

$$
\mathcal{Q}_{j}^{\prime}=t_{j} \star Q_{j}^{\prime}
$$

for integers $t_{j} \geq 1$, and

$$
\chi_{(l)}^{E S}\left(\mathcal{Q}_{j}\right)=\chi_{(l)}^{E S}\left(\mathcal{Q}_{j}^{\prime}\right)
$$

for each $l \leq L$

Proof. For each $j=1, \ldots N$, let $k_{j}$ be the common number of cone points of $Q_{j}$ and $Q_{j}^{\prime}$. Then set

$$
\begin{gathered}
t_{j}=\prod_{i=1, i \neq j}^{N} k_{i}, \\
\mathcal{Q}_{j}=t_{j} \star Q_{j}
\end{gathered}
$$

and

$$
\mathcal{Q}_{j}^{\prime}=t_{j} \star Q_{j}^{\prime}
$$

By Lemma 3.8,

$$
\chi_{(l)}^{E S}\left(\mathcal{Q}_{j}\right)=\chi_{(l)}^{E S}\left(\mathcal{Q}_{j}^{\prime}\right)
$$

for each $l \leq L$. Moreover, each $\mathcal{Q}_{j}$ and $\mathcal{Q}_{j}^{\prime}$ has $t_{j} k_{j}=\prod_{i=1}^{N} k_{i}$ cone points.

In the following lemma, we establish an infinite family of pairs of orbifolds with the same $l$ th Euler-Satake characteristic for $l=0,1,2$ and a number of other properties, each of which being required for constructions in the sequel.

LEMMA 3.10. For each integer $q \geq 2$ and each $g \geq 0$, let

$$
Q[g, q]=\Sigma_{g}\left(2 q+1,2 q+1,2 q^{2}+q\right)
$$

and

$$
Q^{\prime}[g, q]=\Sigma_{g}\left(q+2, q^{2}+2 q, q^{2}+2 q\right)
$$


Then

$$
\chi_{(l)}^{E S}(Q[g, q])=\chi_{(l)}^{E S}\left(Q^{\prime}[g, q]\right)
$$

for $l=0,1,2$. The orbifolds $\left\{Q[g, q], Q^{\prime}[g, q]: g \geq 0, q \geq 2\right\}$ are all distinct. Moreover, if $Q$ and $s \diamond Q$ are elements of $\left\{Q[g, q], Q^{\prime}[g, q]: g \geq 0, q \geq 2\right\}$ for some orbifold $Q$ and integer $s$, then $s=1$.

Proof. Applying Proposition 3.1, for each integer $q \geq 2$ we have

$$
\begin{aligned}
\chi_{(0)}^{E S}(Q[g, q]) & =\frac{1}{q}-1-2 g \\
& =\chi_{(0)}^{E S}\left(Q^{\prime}[g, q]\right), \\
\chi_{(1)}^{E S}(Q[g, q]) & =2-2 g \\
& =\chi_{(1)}^{E S}\left(Q^{\prime}[g, q]\right)
\end{aligned}
$$

and

$$
\begin{aligned}
\chi_{(2)}^{E S}(Q[g, q]) & =1-2 g+5 q+2 q^{2} \\
& =\chi_{(2)}^{E S}\left(Q^{\prime}[g, q]\right) .
\end{aligned}
$$

That these orbifolds are all distinct is obvious; it is impossible that $Q[g, r]=$ $Q^{\prime}[g, q]$, as $Q[g, r]$ has two smaller- and one larger-order cone point, while $Q^{\prime}[g, q]$ has one smaller and two larger. Moreover, $Q[g, r]=Q[g, q]$ implies that $2 r+1=2 q+1$ so that $r=q$, and similarly $Q^{\prime}[g, r]=Q^{\prime}[g, q]$ implies that $r+2=q+2$ so that $r=q$. The remaining claim is clear.

LEMMA 3.11. For each non-negative integer $L$ and any genus $g$, there is a pair of distinct, closed, connected, effective, orientable 2-orbifolds $Q$ and $Q^{\prime}$ with the same number of cone points such that $\chi_{(l)}^{E S}(Q)=\chi_{(l)}^{E S}\left(Q^{\prime}\right)$ for each $l \leq L$. The common genus of $Q$ and $Q^{\prime}$ can be taken to be any non-negative integer $g$. Moreover, if $R$ is any collection of $2^{L-2}$ integers $\geq 2$, then the orders of the cone points of $Q$ and $Q^{\prime}$ can be taken to be elements of the set $\left\{2 q+1,2 q^{2}+q, q+2,2 q+q^{2}: q \in R\right\}$.

Proof. Throughout, we assume all orbifolds have a fixed genus $g$; note that the constructions in this proof hold for any value of $g$.

Let $L \geq 3$ be an integer, and let $q:\left\{1,2, \ldots, 2^{L-2}\right\} \rightarrow\{2,3, \ldots\}$ be the orderpreserving function whose image is $R$; that is, $q\left(j_{1}\right)<q\left(j_{2}\right)$ whenever $j_{1}<j_{2}$. For $j=$ $1, \ldots 2^{L-2}$, let $Q_{j, 2}=Q[g, q(j)]$ and $Q_{j, 2}^{\prime}=Q^{\prime}[g, q(j)]$ be the orbifolds constructed in Lemma 3.10. Here, the subscript 2 indicates that the $Q_{j, 2}$ and $Q_{j, 2}^{\prime}$ have the same $l$ th Euler-Satake characteristic for $l \leq 2$. To summarize what follows, we construct from these $2^{L-2}$ pairs of orbifolds whose $l$ th Euler-Satake characteristics coincide for $l \leq 2$ a collection of $2^{L-3}$ pairs of orbifolds whose $l$ th Euler-Satake characteristics coincide for $l \leq 3$. Continuing recursively, we construct a pair of orbifolds $Q=Q_{1, L}$ and $Q^{\prime}=Q_{1, L}^{\prime}$ whose $l$ th Euler-Satake characteristics coincide for $l \leq L$.

The following describes the recursive step in detail. Let $n \geq 3$ and $1 \leq j \leq 2^{L-n}$ with $j$ odd, and assume that there are orbifolds $Q_{j, n}=\Sigma_{g}\left(\bar{a}_{1}, a_{2}, \ldots, a_{k}\right), Q_{j, n}^{\prime}=$ $\Sigma_{g}\left(b_{1}, b_{2}, \ldots, b_{k}\right), Q_{j+1, n}=\Sigma_{g}\left(c_{1}, c_{2}, \ldots, c_{k}\right)$, and $Q_{j+1, n}^{\prime}=\Sigma_{g}\left(d_{1}, d_{2}, \ldots, d_{k}\right)$ with 
$a_{1}, a_{2}, \ldots, a_{k}, b_{1}, b_{2}, \ldots, b_{k}, c_{1}, c_{2}, \ldots, c_{k}, d_{1}, d_{2}, \ldots, d_{k} \geq 2$ integers such that

$$
\chi_{(l)}^{E S}\left(Q_{j, n}\right)=\chi_{(l)}^{E S}\left(Q_{j, n}^{\prime}\right)
$$

and

$$
\chi_{(l)}^{E S}\left(Q_{j+1, n}\right)=\chi_{(l)}^{E S}\left(Q_{j+1, n}^{\prime}\right)
$$

for each $l=0,1,2, \ldots, n$. Note that this implies that

$$
\sum_{i=1}^{k} a_{i}^{l-1}=\sum_{i=1}^{k} b_{i}^{l-1}
$$

and

$$
\sum_{i=1}^{k} c_{i}^{l-1}=\sum_{i=1}^{k} d_{i}^{l-1}
$$

for each $l=0,1,2, \ldots, n$.

If $\chi_{(n+1)}^{E S}\left(Q_{j, n}\right)=\chi_{(n+1)}^{E S}\left(Q_{j, n}^{\prime}\right)$, then the recursive step is unnecessary for this pair, so set $Q_{(j+1) / 2, n+1}=Q_{j, n}$ and $Q_{(j+1) / 2, n+1}^{\prime}=Q_{j, n}^{\prime}$. Similarly, if $\chi_{(n+1)}^{E S}\left(Q_{j+1, n}\right)=$ $\chi_{(n+1)}^{E S}\left(Q_{j+1, n}^{\prime}\right)$, then set $Q_{(j+1) / 2, n+1}=Q_{j+1, n}$ and $Q_{(j+1) / 2, n+1}^{\prime}=Q_{j+1, n}^{\prime}$. Otherwise, define

$$
\delta_{1}=\sum_{i=1}^{k} a_{i}^{n}-\sum_{i=1}^{k} b_{i}^{n}
$$

and

$$
\delta_{2}=\sum_{i=1}^{k} d_{i}^{n}-\sum_{i=1}^{k} c_{i}^{n}
$$

Note that if $\delta_{1}=0$ then $\chi_{(n+1)}^{E S}\left(Q_{j, n}\right)=\chi_{(n+1)}^{E S}\left(Q_{j, n}^{\prime}\right)$, so we can assume by switching the roles of $Q_{j, n}$ and $Q_{j, n}^{\prime}$ if necessary that $\delta_{1}>0$. Similarly, we assume with no loss of generality that $\delta_{2}>0$.

We construct the orbifolds $Q_{(j+1) / 2, n+1}$ and $Q_{(j+1) / 2, n+1}^{\prime}$ as follows. Let

$$
Q_{(j+1) / 2, n+1}=\left(\delta_{2} \star Q_{j, n}\right) \circledast\left(\delta_{1} \star Q_{j+1, n}\right)
$$

and

$$
Q_{(j+1) / 2, n+1}^{\prime}=\left(\delta_{2} \star Q_{j, n}^{\prime}\right) \circledast\left(\delta_{1} \star Q_{j+1, n}^{\prime}\right) .
$$

That is, $Q_{(j+1) / 2, n+1}$ is given by

$\Sigma_{g}(\overbrace{a_{1}, \ldots, a_{1}}^{\delta_{2}}, \overbrace{a_{2}, \ldots, a_{2}}^{\delta_{2}}, \ldots, \overbrace{a_{k}, \ldots, a_{k}}^{\delta_{2}}, \overbrace{c_{1}, \ldots, c_{1}}^{\delta_{2}}, \overbrace{c_{2}, \ldots, c_{2}}^{\delta_{1}}, \ldots, \overbrace{c_{k}, \ldots, c_{k}}^{\delta_{1}})$, 
and $Q_{(j+1) / 2, n+1}^{\prime}$ is given by

$\Sigma_{g}(\overbrace{b_{1}, \ldots, b_{1}}^{\delta_{2}}, \overbrace{b_{2}, \ldots, b_{2}}^{\delta_{2}}, \ldots, \overbrace{b_{k}, \ldots, b_{k}}^{\delta_{2}}, \overbrace{d_{1}, \ldots, d_{1}}^{\delta_{1}}, \overbrace{d_{2}, \ldots, d_{2}}^{\delta_{1}}, \ldots, \overbrace{d_{k}, \ldots, d_{k}}^{\delta_{1}})$.

Then we can express $\chi_{(n+1)}^{E S}\left(Q_{(j+1) / 2, n+1}\right)-\chi_{(n+1)}^{E S}\left(Q_{(j+1) / 2, n+1}^{\prime}\right)$ as

$$
\begin{array}{r}
\left(2-2 g-\left(\delta_{2} k+\delta_{1} k\right)+\delta_{2} \sum_{i=1}^{k} a_{i}^{n}+\delta_{1} \sum_{i=1}^{k} c_{i}^{n}\right) \\
-\left(2-2 g-\left(\delta_{2} k+\delta_{1} k\right)+\delta_{2} \sum_{i=1}^{k} b_{i}^{n}+\delta_{1} \sum_{i=1}^{k} d_{i}^{n}\right),
\end{array}
$$

yielding

$$
\begin{aligned}
\delta_{2}\left(\sum_{i=1}^{k} a_{i}^{n}-\sum_{i=1}^{k} b_{i}^{n}\right)+\delta_{1}\left(\sum_{i=1}^{k} c_{i}^{n}-\sum_{i=1}^{k} d_{i}^{n}\right) & =\delta_{2} \delta_{1}+\delta_{1}\left(-\delta_{2}\right) \\
& =0 .
\end{aligned}
$$

It follows that

$$
\chi_{(n+1)}^{E S}\left(Q_{(j+1) / 2, n+1}\right)=\chi_{(n+1)}^{E S}\left(Q_{(j+1) / 2, n+1}^{\prime}\right) .
$$

Moreover, for each non-negative integer $l \leq n$, we can express $\chi_{(l)}^{E S}\left(Q_{(j+1) / 2, n+1}\right)-$ $\chi_{(l)}^{E S}\left(Q_{(j+1) / 2, n+1}^{\prime}\right)$ as

$$
\begin{array}{r}
\left(2-2 g-\left(\delta_{2} k+\delta_{1} k\right)+\delta_{2} \sum_{i=1}^{k} a_{i}^{l-1}+\delta_{1} \sum_{i=1}^{k} c_{i}^{l-1}\right) \\
-\left(2-2 g-\left(\delta_{2} k+\delta_{1} k\right)+\delta_{2} \sum_{i=1}^{k} b_{i}^{l-1}+\delta_{1} \sum_{i=1}^{k} d_{i}^{l-1}\right),
\end{array}
$$

resulting in

$$
\delta_{2}\left(\sum_{i=1}^{k} a_{i}^{l-1}-\sum_{i=1}^{k} b_{i}^{l-1}\right)+\delta_{1}\left(\sum_{i=1}^{k} c_{i}^{l-1}-\sum_{i=1}^{k} d_{i}^{l-1}\right)=0
$$

by equations (5) and (6).

For each $n \geq 3$, we apply this construction for each odd $j$ with $1 \leq j \leq 2^{L-n}$, forming $2^{L-n-1}$ orbifold pairs $Q_{(j+1) / 2, n+1}$ and $Q_{(j+1) / 2, n+1}^{\prime}$; note that these are indexed as $Q_{r, n+1}, Q_{r, n+1}^{\prime}$ for $r=1,2, \ldots, 2^{L-n-1}$. For each $r, Q_{r, n+1}$ and $Q_{r, n+1}^{\prime}$ have the same number of cone points; hence, we can apply Lemma 3.9 to the collection of $\left\{Q_{r, n+1}, Q_{r, n+1}^{\prime}: r=1, \ldots, 2^{L-n-1}\right\}$ to assume that they all have the same number of cone points, which is required in the next recursive step. The result is a pair of orbifolds $Q=Q_{1, L}$ and $Q^{\prime}=Q_{1, L}^{\prime}$ with the desired properties. It remains only to show that $Q$ and $Q^{\prime}$ are distinct.

While not all of the $Q_{j, 2}$ and $Q_{j, 2}^{\prime}$ may have been used in this construction (if it happens that $\chi_{(n+1)}^{E S}\left(Q_{j, n}\right)=\chi_{(n+1)}^{E S}\left(Q_{j, n}^{\prime}\right)$ or $\chi_{(n+1)}^{E S}\left(Q_{j+1, n}\right)=\chi_{(n+1)}^{E S}\left(Q_{j+1, n}^{\prime}\right)$ for some $\left.j\right)$, 
but note that both $Q$ and $Q^{\prime}$ have at least three cone points each. Fix the smallest value of $j$ such that $Q$ and $Q^{\prime}$ have cone points arising from $Q[g, q(j)]$ and $Q^{\prime}[g, q(j)]$. While the roles of these two may have switched to ensure that $\delta_{1}$ and $\delta_{2}$ are positive, only one of $Q$ and $Q^{\prime}$ can have cone points of order $q(j)+2$ from $Q^{\prime}[g, q(j)]$. As $q(j) \geq 2$ for all $j$ and $q(j)$ is strictly increasing, it follows that all other cone points of the two orbifolds must have order strictly greater than $q(j)+2$, and hence that $Q$ and $Q^{\prime}$ are distinct orbifolds.

LemMa 3.12. Let L be a non-negative integer. Suppose $Q$ and $Q^{\prime}$ are distinct, closed, connected, effective, orientable 2-orbifolds with the same genus and same number of cone points such that

$$
\chi_{(l)}^{E S}(Q)=\chi_{(l)}^{E S}\left(Q^{\prime}\right)
$$

for $l \leq L$. For any integer $N \geq 2$, there is a collection $\mathcal{Q}_{1}, \mathcal{Q}_{2}, \ldots \mathcal{Q}_{N}$ of distinct closed, effective, orientable 2-orbifolds such that

$$
\chi_{(l)}^{E S}\left(\mathcal{Q}_{1}\right)=\chi_{(l)}^{E S}\left(\mathcal{Q}_{2}\right)=\cdots=\chi_{(l)}^{E S}\left(\mathcal{Q}_{N}\right)
$$

for $l \leq L$. Moreover, the orders of cone points of each $\mathcal{Q}_{j}$ are those of $Q$ and $Q^{\prime}$ only.

Proof. It is obvious that $Q$ and $Q^{\prime}$ must have singular points, as otherwise $\chi_{(0)}^{E S}(Q)=$ $\chi_{(0)}^{E S}\left(Q^{\prime}\right)$ implies that $Q=Q^{\prime}$. By this observation and Lemma 3.5, we may assume without loss of generality that $Q$ has $r$ cone points of order $m$ for some $m \geq 2$, and $Q^{\prime}$ does not have a cone point of order $m$. Let $k$ be the common number of cone points of $Q$ and $Q^{\prime}$.

For $j=1,2, \ldots, N$, we define

$$
\mathcal{Q}_{j}=\overbrace{Q \circledast \cdots \circledast Q}^{N-j} \circledast \overbrace{Q^{\prime} \circledast \cdots \circledast Q^{\prime}}^{j-1} .
$$

Then each $\mathcal{Q}_{j}$ has exactly $(N-j) r$ cone points of order $m$ so that the $\mathcal{Q}_{j}$ are distinct.

Now, let $Q=\Sigma_{g}\left(a_{1}, \ldots, a_{k}\right)$ and $Q^{\prime}=\Sigma_{g}\left(b_{1}, \ldots, b_{k}\right)$ (so that in particular, $a_{i}=m$ for $r$ choices of $i$ ), and note that $\sum_{i=1}^{k} a_{i}^{l-1}=\sum_{i=1}^{k} b_{i}^{l-1}$ for each $l \leq L$. For each $0 \leq l \leq L$ and $1 \leq j \leq N$, we compute

$$
\begin{aligned}
\chi_{(l)}^{E S}\left(\mathcal{Q}_{j}\right) & =2-2 g-(N-1) k+(N-j) \sum_{i=1}^{k} a_{i}^{l-1}+(j-1) \sum_{i=1}^{k} b_{i}^{l-1} \\
& =2-2 g-(N-1) k+(N-j) \sum_{i=1}^{k} a_{i}^{l-1}+(j-1) \sum_{i=1}^{k} a_{i}^{l-1} \\
& =2-2 g-(N-1) k+(N-1) \sum_{i=1}^{k} a_{i}^{l-1} .
\end{aligned}
$$

As $\chi_{(l)}^{E S}\left(\mathcal{Q}_{j}\right)$ does not depend on $j$, we are done.

Proof of Proposition 3.6. By Lemmas 3.10 and 3.11, there exists a pair of orbifolds with the desired properties. By Lemma 3.12, there are $N$ such orbifolds. 
3.3. Negative classification results for general $\Gamma$. Let $\mathfrak{G}$ be a set of finitely generated discrete groups, and let $\mathfrak{A}=\{\Gamma /[\Gamma, \Gamma]: \Gamma \in \mathfrak{G}\}$ denote the collection of abelianizations of elements of $\mathfrak{G}$. Then each $\Gamma /[\Gamma, \Gamma]$ is of the form $\mathbb{Z}^{l} \oplus G$ uniquely for $l \geq 0$ and $G$ finite by the Fundamental Theorem of Finitely Generated Abelian Groups. Let $\mathfrak{F}$ denote the set of $G$ that appear in this decomposition for elements of $\mathfrak{A}$; that is

$$
\mathfrak{F}=\left\{G: \mathbb{Z}^{l} \oplus G \in \mathfrak{A}\right\} .
$$

Let $\mathfrak{P}$ denote the set of primes $p$ such that there is a $G \in \mathfrak{F}$ and $g \in G$ with $|g|$ divisible by $p$. In this section, we prove the following.

THEOREM 3.13. Let $N \geq 2$ be an integer. Let $\mathfrak{G}$ be a non-empty set of finitely generated discrete groups such that the ranks of the elements of $\mathfrak{A}$ are bounded, and $\mathfrak{P}$ is finite. Then there are distinct, closed, connected, effective, orientable 2-orbifolds $Q_{1}, Q_{2}, \ldots, Q_{N}$ such that for each $\Gamma \in \mathfrak{G}$,

$$
\chi_{\Gamma}^{E S}\left(Q_{1}\right)=\chi_{\Gamma}^{E S}\left(Q_{2}\right)=\cdots=\chi_{\Gamma}^{E S}\left(Q_{N}\right) .
$$

The common genus of the $Q_{j}$ can be chosen to be any non-negative integer.

In particular, note that the hypotheses of Theorem 3.13 are obviously satisfied for $\mathfrak{G}$ finite; hence Theorem 1.2 is a trivial consequence. First, we have the following. Recall that an abelian orbifold is an orbifold $Q$ such that every isotropy group of $Q$ is abelian.

LEMMA 3.14. Let $Q$ be an abelian orbifold and $\Gamma$ a finitely generated discrete group. Then $\tilde{Q}_{\Gamma}$ and $\tilde{Q}_{\Gamma /[\Gamma, \Gamma]}$ are diffeomorphic. In particular, if $Q$ is closed, then

$$
\chi_{\Gamma}^{E S}(Q)=\chi_{\Gamma /[\Gamma, \Gamma]}^{E S}(Q) .
$$

Proof. Let $\rho: \Gamma \rightarrow \Gamma /[\Gamma, \Gamma]$ denote the quotient map. For each local group $G_{x}$ of $Q$, it is easy to see that as $G_{x}$ is abelian, the correspondence $\phi_{x} \mapsto \phi_{x} \circ \rho$ is a bijection between $\operatorname{HOM}\left(\Gamma /[\Gamma, \Gamma], G_{x}\right)$ and $\operatorname{HOM}\left(\Gamma, G_{x}\right)$. It clearly follows that

$$
\begin{aligned}
e_{\rho} & : \mathcal{S}_{\mathcal{G}}^{\Gamma /[\Gamma, \Gamma]} \longrightarrow \mathcal{S}_{\mathcal{G}}^{\Gamma} \\
& : \quad \phi_{x} \longmapsto \phi_{x} \circ \rho
\end{aligned}
$$

is a bijective. See [8, Section 3.3] for a more general treatment of maps on sectors induced by group homomorphisms, of which $e_{\rho}$ is an example.

Recall that if $\left\{V_{x}, G_{x}, \pi_{x}\right\}$ is a linear chart for $\mathcal{G}$ at $x$, then $\left\{V_{x}^{\left\langle\phi_{x}\right\rangle}, C_{G_{x}}\left(\phi_{x}\right), \pi_{x}^{\phi_{x}}\right\}$ is a linear chart for $\mathcal{S}_{\mathcal{G}}^{\Gamma}$ at $\phi_{x}$. As, $\operatorname{Im} \phi_{x}=\operatorname{Im} \phi_{x} \circ \rho \leq G_{x}$, it follows that $e_{\rho}$ is simply the identity on charts and hence a $\mathcal{G}$-equivariant diffeomorphism. It hence induces an isomorphism of orbifold groupoids between $\mathcal{G}^{\Gamma}$ and $\mathcal{G}^{\Gamma /[\Gamma, \Gamma]}$.

It follows that, for abelian orbifolds $Q$ and $Q^{\prime}$,

$$
\chi_{\Gamma}^{E S}(Q)=\chi_{\Gamma}^{E S}\left(Q^{\prime}\right) \quad \forall \Gamma \in \mathfrak{G}
$$

if and only if

$$
\chi_{\Lambda}^{E S}(Q)=\chi_{\Lambda}^{E S}\left(Q^{\prime}\right) \quad \forall \Lambda \in \mathfrak{A}
$$


Proof of Theorem 3.13. Suppose $L$ is the maximum rank of the elements of $\mathfrak{A}$. If $\mathfrak{P}$ is empty, then $\mathfrak{A}$ contains only free abelian groups, and the result follows from Proposition 3.6. So assume $\mathfrak{P} \neq \varnothing$.

Let $\mathfrak{P}=\left\{p_{1}, p_{2}, \ldots, p_{r}\right\}$, and define

$$
\begin{aligned}
q:\left\{1,2, \ldots, 2^{L-1}\right\} & \longrightarrow\{2,3, \ldots\} \\
: j & \longmapsto j\left(2 \prod_{i=1}^{r} p_{i}\right)-1
\end{aligned}
$$

Then $q$ is order-preserving, and $q(j) \geq 2$ for each $j$. Moreover, for each $i$ and $j, q(j) \equiv-1$ $\bmod p_{i}$. Hence $2 q(j)+1 \equiv-1 \bmod p_{i}$, and $q(j)+2 \equiv 1 \bmod p_{i}$. It follows that $q(j)$, $2 q(j)+1$, and $q(j)+2$ are not divisible by any element of $\mathfrak{P}$.

By Proposition 3.6, for any choice of genus, there are orbifolds $Q_{1}, \ldots, Q_{N}$ such that $\chi_{(l)}^{E S}\left(Q_{1}\right)=\chi_{(l)}^{E S}\left(Q_{2}\right)=\cdots=\chi_{(l)}^{E S}\left(Q_{N}\right)$ for each $l \leq L$. Moreover, we can choose the $Q_{j}$ so that their cone points all have orders $q(j)+1,2 q(j)^{2}+q(j), q(j)+2,2 q(j)+q(j)^{2}$ for values of $j \in\left\{1,2, \ldots, 2^{L-1}\right\}$; in particular, we use the function $q$ defined above in the proof of Lemma 3.11 .

Fix some $j$, let $q=q(j)$, and let $G \in \mathfrak{F}$. For any homomorphism $\phi: G \rightarrow \mathbb{Z} /(2 q+$ $1) \mathbb{Z}$ and each $g \in G$, the order $|g|$ must be divisible by the order $|\phi(g)|$, which must divide $2 q+1$. However, $|g|$ and $2 q+1$ are relatively prime by construction so that $|\phi(g)|=1$ and $g \in \operatorname{Ker} \phi$. Hence, $\phi$ is the trivial homomorphism. The same argument applies to homomorphisms into $\mathbb{Z} /\left(2 q^{2}+q\right) \mathbb{Z}, \mathbb{Z} /\left(2 q+q^{2}\right) \mathbb{Z}$, and $\mathbb{Z} /(q+2) \mathbb{Z}$.

It follows that for any homomorphism $\phi: \mathbb{Z}^{l} \oplus G \rightarrow \mathbb{Z} / m \mathbb{Z}$, where $m=2 q+$ $1,2 q^{2}+q, 2 q+q^{2}$, or $q+2$, each $g \in G$ is in the kernel, so that

$$
\chi_{\mathbb{Z}^{\prime} \oplus G}^{E S}\left(Q_{j}\right)=\chi_{(l)}^{E S}\left(Q_{j}\right)
$$

for each $\mathbb{Z}^{l} \oplus G \in \mathfrak{A}$ and each $j$, completing the proof.

4. Other classes of orbifolds. In this section, we demonstrate that the hypotheses of Theorem 1.1 cannot be relaxed to include non-effective nor non-orientable orbifolds. Note that in the case of a global quotient, it is convenient to describe the $\Gamma$-sectors globally as originally given in [16]. See equation (1) and [7, Section 3.1] for the equivalence of these definitions.

EXAMPLE 4.1. Let $\mathbb{Z} / 6 \mathbb{Z}=\langle a\rangle$ act on $S^{2}$ so that $a$ acts as a rotation through $\pi / 3$, and let $Q$ denote the resulting quotient orbifold. Then $Q$ is effective, has underlying space homeomorphic to $S^{2}$ and has two cone points, both with isotropy $\mathbb{Z} / 6 \mathbb{Z}$. Similarly, let $\mathbb{Z} / 6 \mathbb{Z}=\langle b\rangle$ act on $S^{2}$ where $b$ acts by a rotation through $2 \pi / 3$. Then the quotient orbifold $Q^{\prime}$ has two cone points with isotropy $\mathbb{Z} / 6 \mathbb{Z}$, and every other point has isotropy $\mathbb{Z} / 3 \mathbb{Z}$. Let $n p, s p \in S^{2}$ denote the two fixed points of each of these actions. We claim that $\chi_{\Gamma}^{E S}(Q)=\chi_{\Gamma}^{E S}\left(Q^{\prime}\right)$ for every finitely generated discrete $\Gamma$.

Let $\iota: a \mapsto b$ denote the obvious isomorphism and fix $\Gamma$ finitely generated and discrete. Then $\phi \mapsto \iota \circ \phi$ of course defines a bijection between $\operatorname{HOM}(\Gamma,\langle a\rangle)$ and $\operatorname{HOM}(\Gamma,\langle b\rangle)$. We note the following.

- If $\operatorname{Im} \phi=\langle 1\rangle$, then $\left(S^{2} ;\langle a\rangle\right)_{(\phi)}=S^{2} \rtimes\langle a\rangle$, diffeomorphic to $Q$, has Euler-Satake characteristic $\frac{1}{3}$ and $\left(S^{2} ;\langle b\rangle\right)_{(\llcorner\circ \phi)}=S^{2} \rtimes\langle b\rangle$, diffeomorphic to $Q^{\prime}$, has Euler characteristic $\frac{1}{3}$. 
- If $\operatorname{Im} \phi=\langle a\rangle$ or $\left\langle a^{2}\right\rangle$, then $\left(S^{2} ;\langle a\rangle\right)_{(\phi)}=\{n p, s p\} \rtimes\langle a\rangle$ has Euler-Satake characteristic $\frac{1}{3}$ and $\left(S^{2} ;\langle a\rangle\right)_{(\llcorner\circ)}=\{n p, s p\} \rtimes\langle b\rangle$ has Euler characteristic $\frac{1}{3}$.

- If $\operatorname{Im} \phi=\left\langle a^{3}\right\rangle$, then $\left(S^{2} ;\langle a\rangle\right)_{(\phi)}=\{n p, s p\} \rtimes\langle a\rangle$ has Euler-Satake characteristic $\frac{1}{3}$ and $\left(S^{2} ;\langle a\rangle\right)_{(เ \circ)}=S^{2} \rtimes\langle b\rangle$, diffeomorphic to $Q^{\prime}$ has Euler characteristic $\frac{1}{3}$.

It follows that

$$
\chi_{E S}\left(\widetilde{(Q)_{(\phi)}}\right)=\chi_{E S}\left(\widetilde{\left.\left(\widetilde{Q}^{\prime}\right)_{(\llcorner\circ \phi)}\right)}\right.
$$

for each $\phi \in \operatorname{HOM}(\Gamma,\langle a\rangle)$, and hence that

$$
\chi_{\Gamma}^{E S}(Q)=\chi_{\Gamma}^{E S}\left(Q^{\prime}\right)
$$

Therefore, there is no finitely generated discrete $\Gamma$ such that $\chi_{\Gamma}^{E S}$ distinguishes between $Q$ and $Q^{\prime}$.

EXAMPLE 4.2. Let $Q$ and $Q^{\prime}$ be orbifolds homeomorphic as topological spaces to the cylinder $S^{1} \times[0,1]$, with $S^{1} \times\{0,1\}$ singular and $S^{1} \times(0,1)$ non-singular as explained below. Let $B_{0}=S^{1} \times\{0\}$ and $B_{1}=S^{1} \times\{1\}$ denote the connected components of the singular set of $Q$, and similarly let $B_{0}^{\prime}$ and $B_{1}^{\prime}$ denote the components of the singular set of of $Q^{\prime}$. Both orbifolds have four corner reflectors as follows, where $D_{2 n}$ denotes the dihedral group of order $2 n$. The orbifold $Q$ has corner reflectors modeled by $\mathbb{R}^{2} / D_{6}$ and $\mathbb{R}^{2} / D_{10}$ on $B_{0}$; and $\mathbb{R}^{2} / D_{14}$ and $\mathbb{R}^{2} / D_{22}$ on $B_{1}$. The orbifold $Q^{\prime}$ has corner reflectors modeled by $\mathbb{R}^{2} / D_{6}$ and $\mathbb{R}^{2} / D_{14}$ on $B_{0}^{\prime}$; and $\mathbb{R}^{2} / D_{10}$ and $\mathbb{R}^{2} / D_{22}$ on $B_{1}^{\prime}$. By examining the components of the singular sets, it is clear that $Q$ and $Q^{\prime}$ are not diffeomorphic.

As all dihedral groups under consideration have an odd number of rotations and hence the centralizer of an element of order 2 is precisely the group generated by that element, it is easy to see that the $\Gamma$-sectors of $Q$ for each finitely generated discrete group $\Gamma$ all occur in the following list:

- an orbifold diffeomorphic to $Q$,

- a circle with trivial $\mathbb{Z} / 2 \mathbb{Z}$-action and

- a point with trivial $\mathbb{Z} / n \mathbb{Z}$-action, where $n=3,5,7$, or 11 .

Similarly, the $\Gamma$-sectors of $Q^{\prime}$ are of the form

- an orbifold diffeomorphic to $Q^{\prime}$,

- a circle with trivial $\mathbb{Z} / 2 \mathbb{Z}$-action and

- a point with trivial $\mathbb{Z} / n \mathbb{Z}$-action, where $n=3,5,7$, or 11 .

There is an obvious bijection between homomorphisms from $\Gamma$ into the local groups of $Q$ and homomorphisms from $\Gamma$ into the local groups of $Q^{\prime}$. This bijection preserves the diffeomorphism class of the corresponding sector in every case except that of the trivial homomorphism, corresponding to the unique sectors diffeomorphic to $Q$ and $Q^{\prime}$. However, by [18, Equation 13.3.4],

$$
\begin{aligned}
\chi_{E S}(Q) & =-\frac{1}{2}\left(4-\frac{1}{3}-\frac{1}{5}-\frac{1}{7}-\frac{1}{11}\right) \\
& =\frac{-1867}{1155} \\
& =\chi_{E S}\left(Q^{\prime}\right),
\end{aligned}
$$

and it follows that $\chi_{\Gamma}^{E S}(Q)=\chi_{\Gamma}^{E S}\left(Q^{\prime}\right)$ for every finitely generated discrete $\Gamma$. 
Finally, we note that the constructions of orbifolds whose $\Gamma$-Euler-Satake characteristics coincide given above can be used to construct orbifolds of arbitrary even dimension with the same properties.

Corollary 4.3. Let $N, n \geq 2$ be integers withn even. Let $\mathfrak{G}$ be anon-empty collection of finitely generated discrete groups such that, with the notation as in Section 3.3, the ranks of the elements of $\mathfrak{A}$ are bounded, and $\mathfrak{P}$ is finite. Then there are distinct closed, connected, effective, orientable n-dimensional orbifolds $Q_{1}, Q_{2}, \ldots, Q_{N}$ such that for each $\Gamma \in \mathfrak{G}$,

$$
\chi_{\Gamma}^{E S}\left(Q_{1}\right)=\chi_{\Gamma}^{E S}\left(Q_{2}\right)=\cdots=\chi_{\Gamma}^{E S}\left(Q_{N}\right)
$$

Proof. Since the $\Gamma$-Euler-Satake characteristic is multiplicative (see $[8$, Section 4.1]), we need only apply Theorem 3.13 and take the product of each 2-orbifold with $S^{n-2}$.

ACKNOWLEDGEMENTS. This paper is the result of the course 'Topics: Orbifold Euler Characteristics' taught in the Rhodes College Mathematics and Computer Science Department in the fall of 2008. We would like to express our appreciation to the department and college for the versatility and support that allowed us to hold this seminar and explore these results. We would also like to thank Rachel Dunwell for helpful suggestions and assistance. Additionally, we would like to thank the referee for helpful comments and corrections.

The third author would like to thank Carla Farsi and Anna Casteen, with whom he has conducted work leading to this project. In particular, Proposition 3.1 was first proved by Anna Casteen as part of her senior seminar project 'Finding orbifold Euler characteristics' at Rhodes College in the spring of 2008.

\section{REFERENCES}

1. A. Adem, J. Leida and Y. Ruan, Orbifolds and stringy topology, Cambridge Tracts in Mathematics 171 (Cambridge University Press, Cambridge, UK, 2007).

2. M. Boileau, S. Maillot and J. Porti, Three-Dimensional Orbifolds and their Geometric Structures, Panoramas et Synthéses, 15 (Société Mathématique de France, Paris, 2003).

3. J. Bryan and J. Fulman, Orbifold Euler characteristics and the number of commuting $m$-tuples in the symmetric groups, Ann. Comb. 2(1) (1998), 1-6.

4. W. Chen and Y. Ruan, Orbifold Gromov-Witten theory, in Orbifolds in mathematics and physics (Madison, WI, 2001), Contemp. Math., 310, Amer. Math. Soc. (Providence, RI, 2002), 25-85.

5. W. Chen and Y. Ruan, A new cohomology theory of orbifold, Commun. Math. Phys. 248(1) (2004), 1-31.

6. L. Dixon, J. Harvey, C. Vafa and E. Witten, Strings on orbifolds, Nucl. Phys. B 261(4) (1985), 678-686.

7. C. Farsi and C. Seaton, Generalized twisted sectors of orbifolds, Pacific J. Math. 246(1) (2010), 49-74.

8. C. Farsi and C. Seaton, Generalized orbifold Euler characteristics for general orbifolds and wreath products, arXiv:0902.1198v1 [math.DG] (2009).

9. C. Farsi and C. Seaton, Nonvanishing vector fields on orbifolds, Trans. Amer. Math. Soc. 362(1) (2010), 509-535.

10. T. Kawasaki, The signature theorem for $V$-manifolds, Topology 17(1) (1978), 75-83.

11. W. S. Massey, Algebraic topology: An introduction, Graduate Texts in Mathematics 56 (Springer, Berlin, 1984). 
12. I. Moerdijk and J. Mrčun, Introduction to Foliations and Lie Groupoids, Cambridge Studies in Advanced Mathematics 91 (Cambridge University Press, Cambridge, UK, 2003).

13. I. Moerdijk and D. Pronk, Simplicial cohomology of orbifolds, Indag. Math. (N.S.) 10(2) (1999), 269-293.

14. S. S. Roan, Minimal resolutions of Gorenstein orbifolds in dimension three, Topology 35(2) (1996), 489-508.

15. I. Satake, The Gauss-Bonnet theorem for $V$-manifolds, J. Math. Soc. Japan 9 (1957), 464-492.

16. H. Tamanoi, Generalized orbifold Euler characteristic of symmetric products and equivariant Morava K-theory, Algebr. Geom. Topol. 1 (2001), 115-141.

17. H. Tamanoi, Generalized orbifold Euler characteristic of symmetric orbifolds and covering spaces, Algebr. Geom. Topol. 3 (2003), 791-856.

18. W. Thurston, The Geometry and Topology of 3-Manifolds, Lecture Notes (Princeton University Mathematics Deptment, Princeton, NJ, 1978). 